\title{
Topological superfluids on a square optical lattice with non-Abelian gauge fields: Effects of next-nearest-neighbor hopping in the BCS-BEC evolution
}

\author{
M. Iskin \\ Department of Physics, Koç University, Rumelifeneri Yolu, 34450 Sartyer, Istanbul, Turkey
}

(Received 28 September 2015; published 12 January 2016)

\begin{abstract}
We consider a two-component Fermi gas with attractive interactions on a square optical lattice, and study the interplay of Zeeman field, spin-orbit coupling, and next-nearest-neighbor hopping on the ground-state phase diagrams in the entire BCS-BEC evolution. In particular, we first classify and distinguish all possible superfluid phases by the momentum-space topology of their zero-energy quasiparticle-quasihole excitations, and then numerically establish a plethora of quantum phase transitions in between. These transitions are further signaled and evidenced by the changes in the corresponding topological invariant of the system, i.e., its Chern number. Lastly, we find that the superfluid phase exhibits a reentrant structure, separated by a fingering normal phase, the origin of which is traced back to the changes in the single-particle density of states.
\end{abstract}

DOI: 10.1103/PhysRevA.93.013608

\section{INTRODUCTION}

In an archetypal square optical lattice [1], since either the lattice potential is separable in $x$ and $y$ directions, and therefore, the atoms are strictly not allowed to tunnel (hop) along the diagonal, i.e., the next-nearest neighbor (NNN), directions, or the diagonal hoppings are negligible, the singleparticle dynamics is controlled by the nearest-neighbor (NN) hoppings. However, it has been recently argued that the ratio of NNN to NN hopping can be effectively tuned all the way from 0 to $\infty$ in driven optical lattices, by periodically shaking the lattice potential in time, e.g., see [2,3]. Indeed, pioneering experiments on such lattices have already paved the way towards "tunnelling engineering," allowing for tunable (real and/or complex) NN and NNN hoppings [4-6]. For instance, applying similar techniques to a honeycomb optical lattice, and by controlling not only the amplitude but also the phase of the complex NNN tunneling [8], the ETH Zurich group has recently reported the very first realization of the topological Haldane model [7] with ultracold fermions.

In addition to the driven optical lattices, other methods relying on the laser-assisted tunneling and potential energy gradient have also been used to engineer spatially dependent complex hoppings, by coupling the internal (atomic) and external (space) degrees of freedom, which are analogous to having artificial gauge fields $[9,10]$. The recent realizations of the Hofstadter-Harper model are some of the prime examples $[11,12]$. Indeed, the fruitful quest for creating various Abelian or non-Abelian artificial gauge fields in discrete (lattice) and continuum systems has been pursued by many groups in the past decade or so, and there are numerous ultracold realizations including the spin-orbit coupling (SOC) [13-18].

Motivated by the advent of driven optical lattices with tunable hoppings, here we consider a two-component Fermi gas with attractive interactions on a square optical lattice, and study the interplay of Zeeman field, SOC, and NNN hopping on the ground-state phase diagrams in the entire BCS-BEC evolution. In particular, we firmly establish a great deal of quantum phase transitions between superfluid (SF) phases with distinct momentum (k) space topologies, that are driven mainly by the Zeeman field. In addition, we also derive analytical expressions for the changes in the Chern number $(\mathrm{CN})$ showing that these phase transitions are further signaled and evidenced by the changes in the topological invariant of the system when there is SOC. Furthermore, we find that the SF phase exhibits a reentrant structure, separated by a fingering normal phase, as a function of total particle filling. This intricate structure is a result of combined SOC and NNN hopping, and we trace its origin back to the single-particle density of states (DoS) of the system which is shown to exhibit a number of narrow strips (depending on the SOC strength) as a function of energy.

The rest of the paper is organized as follows. In Sec. II, we first introduce the model Hamiltonian, then discuss the effects of SOC both on the single-particle problem and on the corresponding DoS, and then derive the self-consistency mean-field equations for handling the many-body problem. In Sec. III, we first classify and distinguish all possible SF phases by the $\mathbf{k}$-space topology of their zero-energy quasiparticlequasihole excitations, and then derive the corresponding changes in the $\mathrm{CN}$ of the system. Our numerical calculations and the resultant ground-state phase diagrams are presented and thoroughly analyzed in Sec. IV, and the paper ends with a brief summary of our conclusions and an outlook in Sec. V.

\section{MEAN-FIELD THEORY}

To explore the possible quantum phases of spin-orbit coupled Fermi gases loaded into the periodic arrays of an optical lattice potential, we limit our discussion to the tight-binding atom-hopping regime. The single-band Hubbard model description is known to serve quite well in this regime, for which the gauge fields can be taken into account via the Peierls substitution. In particular, we study the topological effects of a generic non-Abelian gauge field [19],

$$
\mathbf{A}=\left(\alpha \sigma_{y},-\beta \sigma_{x}\right)
$$

on two-dimensional lattice Fermi gases, where $\sigma_{x}$ and $\sigma_{y}$ are the Pauli-spin matrices, and the parameters $\alpha \geqslant 0$ and $\beta \geqslant 0$ characterize both the strength and the symmetry of the SOC. Since the atom-atom interactions are extremely short ranged in a typical cold-atom setting, the interactions are assumed to be onsite and attractive, and we treat such an interaction term in the Hamiltonian with the BCS mean-field approach for the entire BCS-BEC evolution. It is well accepted in the cold-atom 
community that similar treatments for related problems have provided qualitatively correct descriptions of atomic systems at least for their ground states.

\section{A. Model Hamiltonian}

As a result, we study ground-state phases of the following Hamiltonian:

$$
\begin{aligned}
H= & -\sum_{\sigma \sigma^{\prime} i j} c_{\sigma^{\prime} j}^{\dagger} t_{j i}^{\sigma^{\prime} \sigma} c_{\sigma i}-h \sum_{i}\left(c_{\uparrow i}^{\dagger} c_{\uparrow i}-c_{\downarrow i}^{\dagger} c_{\downarrow i}\right) \\
& +\sum_{i}\left(\Delta_{i} c_{\uparrow i}^{\dagger} c_{\downarrow i}^{\dagger}+\Delta_{i}^{*} c_{\downarrow i} c_{\uparrow i}+\frac{\left|\Delta_{i}\right|^{2}}{g}\right) \\
& -\mu \sum_{\sigma i} c_{\sigma i}^{\dagger} c_{\sigma i},
\end{aligned}
$$

where the operator $c_{\sigma i}^{\dagger}\left(c_{\sigma i}\right)$ creates (annihilates) a pseudospin $\sigma=\{\uparrow, \downarrow\}$ fermion at lattice site $i$ [20]. In the presence of a gauge field, the hopping of atoms from site $i$ to $j$ can be described in general by $t_{j i}^{\sigma^{\prime} \sigma}=t_{j i} e^{-\mathrm{i} \theta_{j i}^{\sigma^{\prime} \sigma}}$, where $t_{j i}$ is its amplitude and the accumulated phase factor $\theta_{j i}=\int_{\mathbf{r}_{\mathbf{i}}}^{\mathbf{r}_{\mathbf{j}}} \mathbf{A} \cdot d \mathbf{r}$ is a consequence of the Peierls substitution with $\mathbf{r}_{\mathbf{i}}$ the position of site $i$. The lattice spacing $a$ is set to unity throughout the paper. In the pairing terms, the complex number $\Delta_{i}=g\left\langle c_{\uparrow i} c_{\downarrow i}\right\rangle$ is the local SF order parameter for the mean-field ground state, where $g \geqslant 0$ is the strength of the onsite attraction between $\uparrow$ and $\downarrow$ fermions, and $\langle\cdots\rangle$ is a thermal average. Lastly, the chemical potential $\mu$ determines the total number $N=N_{\uparrow}+N_{\downarrow}$ of atoms where $N_{\sigma}=\sum_{i} n_{\sigma i}$ with the local fermion filling $0 \leqslant n_{\sigma i}=\left\langle c_{\sigma i}^{\dagger} c_{\sigma i}\right\rangle \leqslant 1$, and the out-of-plane Zeeman field $h \geqslant 0$ determines the polarization $P=\left(N_{\uparrow}-N_{\downarrow}\right) / N \geqslant 0$ of the system which is assumed to be positive without losing generality.

Having a translationally invariant lattice in mind, this Hamiltonian can be easily solved using the Fourier series expansion of the annihilation operator $c_{\sigma i}=$ $(1 / \sqrt{M}) \sum_{\mathbf{k}} e^{\mathbf{i k} \cdot \mathbf{r}_{\mathbf{i}}} c_{\sigma \mathbf{k}}$ in momentum space and its Hermitian conjugate, where $M \rightarrow \infty$ is the number of lattice sites in the system and $c_{\sigma \mathbf{k}}$ annihilates a $\sigma$ fermion with wave vector $\mathbf{k}=\left(k_{x}, k_{y}\right)$. For instance, the single-particle terms can be compactly written in this representation as $H_{0}=$ $\sum_{\sigma \sigma^{\prime} \mathbf{k}} c_{\sigma^{\prime} \mathbf{k}}^{\dagger} h_{0 \mathbf{k}}^{\sigma^{\prime} \sigma} c_{\sigma \mathbf{k}}$, where the $2 \times 2$ matrix $h_{0 \mathbf{k}}^{\sigma^{\prime} \sigma}=\epsilon_{\mathbf{k}} \sigma_{0}-$ $h \sigma_{z}+\mathbf{S}_{\mathbf{k}} \cdot \vec{\sigma}$ describes the Hamiltonian dynamics. Here, $\sigma_{0}$ is the identity matrix, $\vec{\sigma}=\left(\sigma_{x}, \sigma_{y}, \sigma_{z}\right)$ is a vector of spin matrices, $\epsilon_{\mathbf{k}}$ is the energy dispersion, and $\mathbf{S}_{\mathbf{k}}=\left(S_{\mathbf{k}}^{x}, S_{\mathbf{k}}^{y}, 0\right)$ is the spin-momentum coupling (i.e., SOC). The specific forms of $\epsilon_{\mathbf{k}}$ and $\mathbf{S}_{\mathbf{k}}$ depend on the particular lattice geometry of interest.

\section{B. Square lattice with $\mathrm{NN}$ and NNN hoppings}

In this paper, we consider a square crystal lattice with primitive unit vectors $\mathbf{a}_{1}=(1,0)$ and $\mathbf{a}_{2}=(0,1)$, and allow for $\mathrm{NN}$ as well as NNN hoppings and set all of the longer-ranged hoppings to zero for simplicity. While $t_{j i}=t \geqslant 0$ is assumed to be positive for NN hoppings, we vary both the strength and the sign of NNN hoppings $t_{j i}=t^{\prime}$. Therefore, the $\uparrow$ and $\downarrow$ atoms gain $\theta_{i+\widehat{\mathbf{x}}, i} \rightarrow e^{-\mathrm{i} \alpha \sigma_{y}}$ factors for NN hopping in the positive $\widehat{\mathbf{x}}$ direction, $\theta_{i+\widehat{\mathbf{y}}, i} \rightarrow e^{\mathrm{i} \beta \sigma_{x}}$ factors for NN hopping in the positive $\widehat{\mathbf{y}}$ direction, and $\theta_{i+\widehat{\mathbf{e}}_{+}, i} \rightarrow e^{-\mathrm{i}\left( \pm \alpha \sigma_{y}-\beta \sigma_{x}\right)}$ factors for
NNN hoppings in the diagonal $\widehat{\mathbf{e}}_{ \pm}=( \pm \widehat{\mathbf{x}}+\widehat{\mathbf{y}}) / \sqrt{2}$ directions. The phase factors for hoppings in the opposite $(-\widehat{\mathbf{x}},-\widehat{\mathbf{y}}$, and $\left.-\widehat{\mathbf{e}}_{ \pm}\right)$directions are given by the corresponding Hermitian conjugates. Using the identity $e^{\mathrm{i} \mathbf{v} \cdot \vec{\sigma}}=\sigma_{0} \cos v+i(\widehat{\mathbf{v}} \cdot \vec{\sigma}) \sin v$ valid for any given vector $\mathbf{v}=v \widehat{\mathbf{v}}$, we obtain

$$
\begin{gathered}
\epsilon_{\mathbf{k}}=-2 t \cos \alpha \cos k_{x}-2 t \cos \beta \cos k_{y} \\
-4 t^{\prime} \cos \gamma \cos k_{x} \cos k_{y}, \\
S_{\mathbf{k}}^{x}=-2 t \sin \beta \sin k_{y}-4 t^{\prime} \beta \frac{\sin \gamma}{\gamma} \cos k_{x} \sin k_{y}, \\
S_{\mathbf{k}}^{y}=2 t \sin \alpha \sin k_{x}+4 t^{\prime} \alpha \frac{\sin \gamma}{\gamma} \sin k_{x} \cos k_{y},
\end{gathered}
$$

where $\gamma=\sqrt{\alpha^{2}+\beta^{2}}$. Note that the reciprocal of a square lattice is also a square lattice in $\mathbf{k}$ space with primitive unit vectors $\mathbf{b}_{1}=(2 \pi, 0)$ and $\mathbf{b}_{2}=(0,2 \pi)$, and therefore, the first $\mathrm{BZ}$ is bounded by $\left|k_{x}\right|=\pi$ and $\left|k_{y}\right|=\pi$.

\section{Helicity bands}

To isolate the effects of SOC on the single-particle problem, we set $h=0$ and analyze the resultant band structures in the first BZ. When $h=0$, the eigenvalues $\epsilon_{\mathbf{k}} \pm\left|S_{\mathbf{k}}\right|$ of the noninteracting Hamiltonian matrix $h_{0 \mathbf{k}}$ correspond, respectively, to the single-particle excitation energies for the positive and negative helicity branches.

In the absence of an SOC, the positive and negative helicity bands are trivially degenerate (identical) everywhere in $\mathbf{k}$ space. However, the presence of an SOC lifts most of this degeneracy, except for five sets of touching points determined by the condition $\left|S_{\mathbf{k}}\right|=0$. In addition to the center $\mathbf{k}_{\mathbf{1}}=(0,0)$ of the square-shaped BZ, the two-point set $\mathbf{k}_{\mathbf{2}}=(0, \pm \pi)$ corresponds to the midpoints of the top and bottom edges of the BZ adding in total to a one full point, the two-point set $\mathbf{k}_{\mathbf{3}}=( \pm \pi, 0)$ corresponds to the midpoints of the right and left edges of the $\mathrm{BZ}$ adding in total to a one full point, the four-point set $\mathbf{k}_{\mathbf{4}}=( \pm \pi, \pm \pi)$ corresponds to the four corners of the BZ adding again to a one full point, and the four-point set $\mathbf{k}_{\mathbf{5}}=\left(k_{5}^{x}, k_{5}^{y}\right)$ is such that $\cos k_{5}^{x}=$ $-\frac{\sin \beta}{2 \beta} \frac{\gamma}{\sin \gamma} \frac{t}{t^{\prime}}$ and $\cos k_{5}^{y}=-\frac{\sin \alpha}{2 \alpha} \frac{\gamma}{\sin \gamma} \frac{t}{t^{\prime}}$. Therefore, while the locations of $\mathbf{k}_{\mathbf{1}}, \mathbf{k}_{\mathbf{2}}, \mathbf{k}_{\mathbf{3}}$, and $\mathbf{k}_{\mathbf{4}}$ points are independent of $t^{\prime}$, the last set $\mathbf{k}_{\mathbf{5}}$ exists beyond a critical $t^{\prime}$ value determined by $\left|t^{\prime}\right|>\frac{\gamma}{\sin \gamma} \frac{\sin \beta}{2 \beta} t$ and $\left|t^{\prime}\right|>\frac{\gamma}{\sin \gamma} \frac{\sin \alpha}{2 \alpha} t$, the minimum of which occurs in the $(\alpha, \beta) \rightarrow(0,0)$ limit where $\left|t^{\prime}\right| \rightarrow t / 2$. The corresponding energy dispersions at the degenerate points are $\epsilon_{\mathbf{k}_{1}}=-2 t(\cos \alpha+\cos \beta)-4 t^{\prime} \cos \gamma$ for the first set, $\epsilon_{\mathbf{k}_{2}}=-2 t(\cos \alpha-\cos \beta)+4 t^{\prime} \cos \gamma$ for the second set, $\epsilon_{\mathbf{k}_{3}}=2 t(\cos \alpha-\cos \beta)+4 t^{\prime} \cos \gamma$ for the third set, $\epsilon_{\mathbf{k}_{4}}=$ $2 t(\cos \alpha+\cos \beta)-4 t^{\prime} \cos \gamma$ for the fourth set, and $\epsilon_{\mathbf{k}_{\mathbf{5}}}=$ $\frac{t^{2}}{t^{\prime}}\left(\cos \alpha \frac{\sin \beta}{\beta}+\cos \beta \frac{\sin \alpha}{\alpha}-\frac{\sin \beta}{\beta} \frac{\sin \alpha}{\alpha} \frac{\gamma}{\tan \gamma}\right) \frac{\gamma}{\sin \gamma}$ for the last set.

For instance, in Fig. 1, we show typical band structures as a function of $\mathbf{k}$ within the first BZ for (a) $t^{\prime} / t=0$, (b) 0.5 , (c) 1 , and (d) 2 , when $\alpha=\beta=\pi / 4$. Here, since the critical threshold is $t^{\prime} \approx 0.56 t$, the fifth degenerate set clearly emerges only in the latter two figures, where $k_{5}^{x}=k_{5}^{y}$ is approximately given by $0.69 \pi$ and $0.59 \pi$ when $t^{\prime} / t$ is 1 and 2 , respectively. When $t^{\prime}$ is near the threshold value as shown in Fig. 1(b), we 
(a)

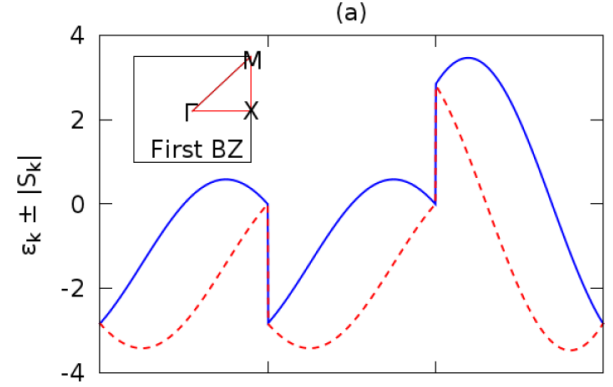

(b)

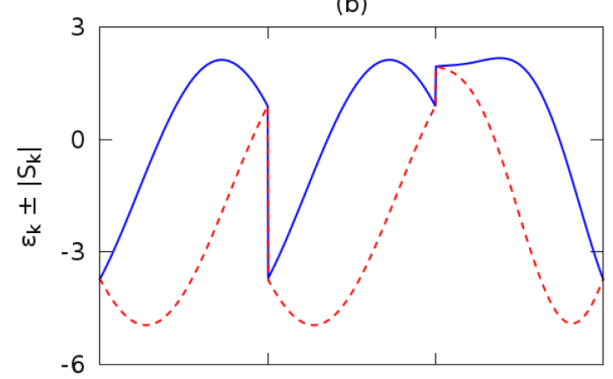

(c)

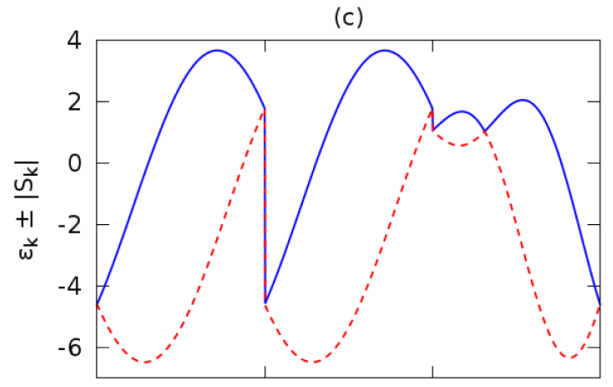

(d)

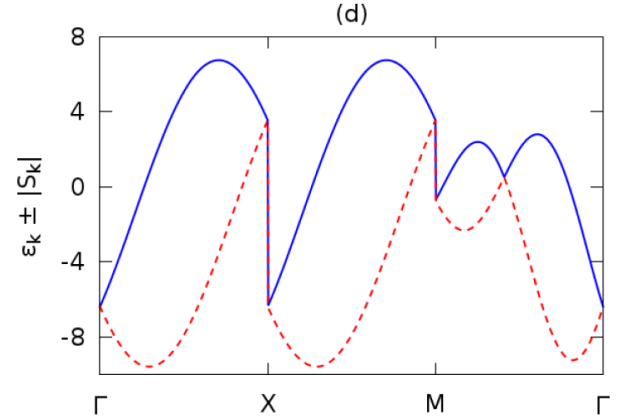

FIG. 1. The positive (straight) and negative (dashed) helicity bands $\epsilon_{\mathbf{k}} \pm\left|S_{\mathbf{k}}\right|$ are shown in units of $t$ as a function of momentum $\mathbf{k}$ within the first BZ. Here, $\Gamma, \mathrm{X}$, and $\mathrm{M}$ correspond, respectively, to $(0,0),(\pi, 0)$, and $(\pi, \pi)$ points, where (a) $t^{\prime} / t=0$, (b) 0.5 , (c) 1 , and (d) 2 , and $\alpha=\beta=\pi / 4$ in all figures.

note that the positive branch becomes almost flat in a sizeable $\mathbf{k}$-space region close to the $\mathrm{M}$ point along the $\Gamma$ direction. More importantly, we also note that the bands depend linearly on $\mathbf{k}$ in the vicinity of all of the touching points, and therefore, they play vital roles in the many-particle problem. This is a direct consequence of enhanced single-particle density of states by SOC as illustrated next for a number of $(\alpha, \beta)$ values.

\section{Density of states (DoS)}

The total $\operatorname{DoS} D(\omega)=D_{+}(\omega)+D_{-}(\omega)$ is given by $D_{ \pm}(\omega)=(1 / M) \sum_{\mathbf{k}} \delta\left(\omega-\epsilon_{\mathbf{k}} \mp\left|S_{\mathbf{k}}\right|\right)$, where $\delta(x) \quad$ is the Dirac-delta function, and the following symmetries $D\left(\omega, t^{\prime}\right)=D\left(-\omega,-t^{\prime}\right)$ and $D_{-}\left(\omega, t^{\prime}\right)=D_{+}\left(-\omega,-t^{\prime}\right)$ hold. We use the broadened definition of the delta function $\pi \delta(x) \rightarrow \eta /\left(x^{2}+\eta^{2}\right)$ in our numerical calculations with $\eta=0.01 t$, which is known to be one of the exact representations of $\delta(x)$ when $\eta \rightarrow 0$.

In Fig. 2, we show colored maps of $t D(\omega)$ as functions of $\omega / t$ and $t^{\prime} / t$ for various $(\alpha, \beta)$ values. In the first five figures, we study the effects of SOC strength by varying $\alpha=\beta$ as (a) 0 , (b) $\pi / 8$, (c) $\pi / 4$, (d) $\pi / 3$, and (e) $\pi / 2$. In the absence of an SOC, shown in Fig. 2(a), the peak position of $D(\omega)$ first shifts from $\omega=0$ to finite values as $\left|t^{\prime}\right|$ increases from 0 to $0.5 t$, and then it gradually returns back to $\omega=0$ in the $\left|t^{\prime}\right| \gg t$ limit. In addition, the diverging peak of $D(\omega)$ is considerably broadened by finite $t^{\prime}$ especially around $\left|t^{\prime}\right| \sim t / 2$. Therefore, $D(\omega)$ is asymmetric around $\omega=0$ for any given $t^{\prime} \neq 0$ due to the broken particle-hole symmetry by the NNN hopping. On the other hand, in the absence of an NNN hopping, while a weak SOC splits the peak of $D(\omega)$ symmetrically around $\omega=0$ as shown in Fig. 2(b), a strong SOC causes two additional peaks located at the bottom and top of the spectrum as shown in Figs. 2(c) and 2(d). This is a consequence of the broken inversion symmetry by the SOC. At the maximally attainable SOC shown in Fig. 2(e), $D(\omega)$ has two isolated and broad peaks that are separated by an energy gap at $\omega=0$. When both SOC and NNN hopping are coupled, $D(\omega)$ shows a peculiar dependence on $\omega$ as shown in Figs. 2(b)-2(e). For completeness, we show the effects of the symmetry of SOC in the remaining three figures, where we set $\alpha=\pi / 4$ and vary $\beta$ as (f) $\pi / 8$, (g) $\pi / 3$, and (h) $\pi / 2$. Note that when $\alpha$ or $\beta$ is strictly zero, the SOC can be gauged away, i.e., their $D(\omega)$ are identical to Fig. 2(a).

Having introduced the model Hamiltonian and discussed the effects of SOC both on the single-particle problem and on the corresponding DoS, next we briefly go over the details of the theoretical formalism for handling the many-body problem.

\section{E. BCS-BEC evolution}

In the absence of a gauge field, it has been well documented in the recent literature that the mean-field description of the SF phases given in Eq. (2) not only works in the BCS and BEC limits but it also captures much of the ground-state properties in the entire BCS-BEC evolution even in two dimensions [21-24]. Motivated by the success of this description in related problems, here we apply it to investigate the fate of $\mathrm{SF}$ phases under a generic non-Abelian gauge field given by Eq. (1).

For the many-body problem, the k-space representation again proves to be more convenient, in which case the meanfield Hamiltonian can be compactly written as

$$
\begin{aligned}
H= & \frac{1}{2} \sum_{\mathbf{k}} \psi_{\mathbf{k}}^{\dagger}\left(\begin{array}{cccc}
\xi_{\mathbf{k}}-h & S_{\mathbf{k}}^{\perp} & 0 & \Delta \\
S_{\mathbf{k}}^{\perp *} & \xi_{\mathbf{k}}+h & -\Delta & 0 \\
0 & -\Delta^{*} & -\xi_{\mathbf{k}}+h & S_{\mathbf{k}}^{\perp *} \\
\Delta^{*} & 0 & S_{\mathbf{k}}^{\perp} & -\xi_{\mathbf{k}}-h
\end{array}\right) \psi_{\mathbf{k}} \\
& +\sum_{\mathbf{k}} \xi_{\mathbf{k}}+M \frac{|\Delta|^{2}}{g},
\end{aligned}
$$


(a)

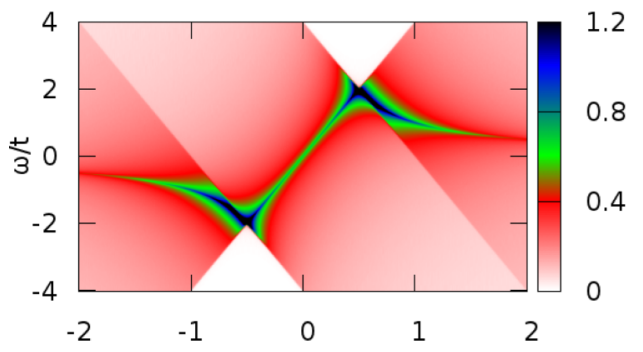

(c)

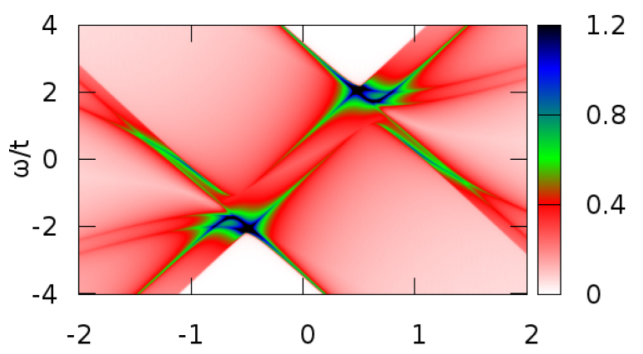

(e)

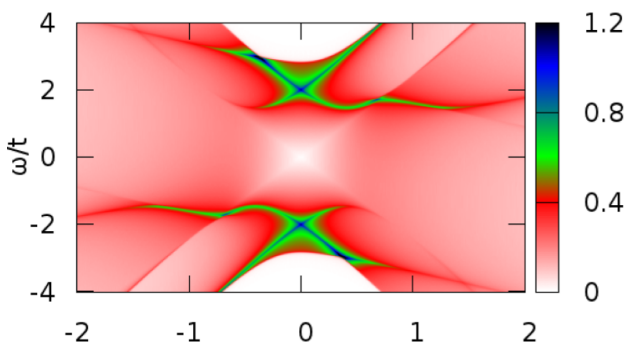

(g)

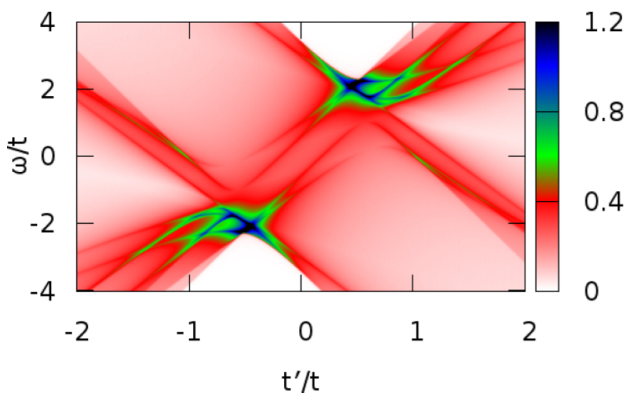

(b)

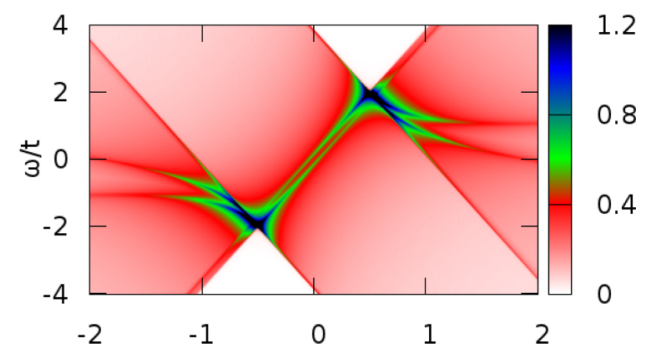

(d)

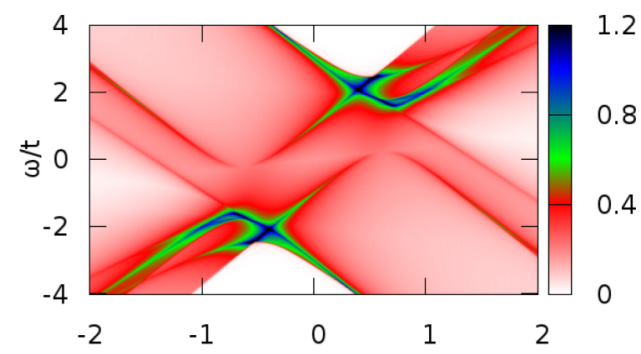

(f)

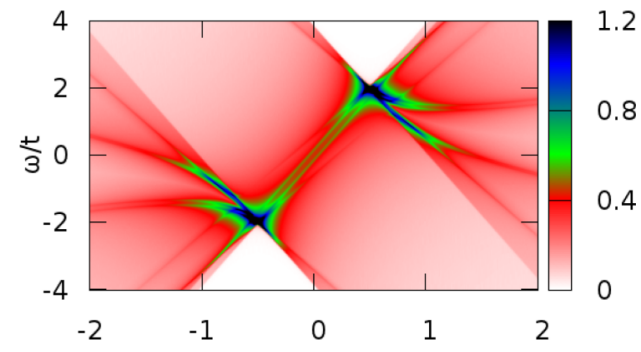

(h)

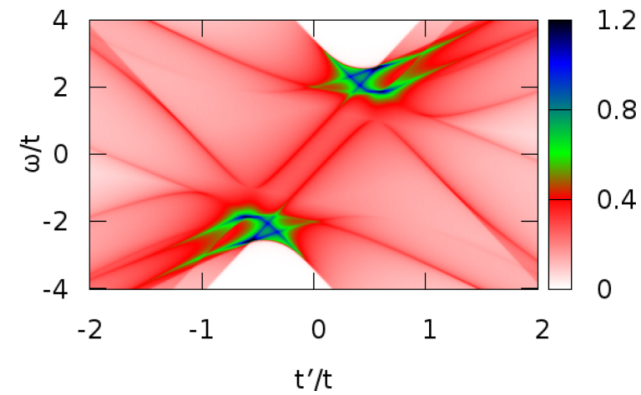

FIG. 2. The colored maps of the total $\operatorname{DoS} D(\omega)$ are shown in units of $1 / t$ and as functions of energy $\omega / t$ and NNN hopping $t^{\prime} / t$ for a number of $(\alpha, \beta)$ values. In the first five figures, we vary $\alpha=\beta$ as (a) $\rightarrow 0^{+}$, (b) $\pi / 8$, (c) $\pi / 4$, (d) $\pi / 3$, and (e) $\pi / 2$. In the remaining three figures, we set $\alpha=\pi / 4$ and vary $\beta$ as (f) $\pi / 8,(\mathrm{~g}) \pi / 3$, and (h) $\pi / 2$. Note that when $\alpha$ or $\beta$ is strictly zero, the SOC can be gauged away, i.e., their $D(\omega)$ are identical to (a).

where the operator $\psi_{\mathbf{k}}^{\dagger}=\left[c_{\uparrow \mathbf{k}}^{\dagger}, c_{\downarrow \mathbf{k}}^{\dagger}, c_{\uparrow,-\mathbf{k}}, c_{\downarrow,-\mathbf{k}}\right]$ denotes the creation and annihilation operators collectively. The $4 \times 4$ matrix seen in Eq. (6) is the Hamiltonian matrix, say $\mathbf{D}_{\mathbf{k}}$, and it involves the shifted dispersion $\xi_{\mathbf{k}}=\epsilon_{\mathbf{k}}-\mu$, SOC $S_{\mathbf{k}}^{\perp}=S_{\mathbf{k}}^{x}-\mathrm{i} S_{\mathbf{k}}^{y}$, and complex order parameter $\Delta$ describing the pairing of $\uparrow$ and $\downarrow$ atoms in $\mathbf{k}$ space with zero center-of-mass momentum. Note that $\Delta=g \sum_{\mathbf{k}}\left\langle c_{\uparrow \mathbf{k}} c_{\downarrow,-\mathbf{k}}\right\rangle$ is uniform in $\mathbf{k}$ space since we allow only for onsite atom-atom interactions in real space. The mean-field thermodynamic potential for such an $H$ can be written in general as

$$
\Omega=\frac{T}{2} \sum_{\lambda \mathbf{k}} \ln \left(\frac{1+X_{\lambda \mathbf{k}}}{2}\right)+\sum_{\mathbf{k}} \xi_{\mathbf{k}}+M \frac{|\Delta|^{2}}{g},
$$

where $T$ is the temperature, $\lambda=\{1,2,3,4\}$ labels the eigenvalues $E_{\lambda \mathbf{k}}$ of the Hamiltonian matrix (i.e., the quasiparticlequasihole excitation energies) and $X_{\lambda \mathbf{k}}=\tanh \left[E_{\lambda \mathbf{k}} /(2 T)\right]$. The Boltzmann constant $k_{B}$ is set to unity throughout this paper. The eigenvalues of our $\mathbf{D}_{\mathbf{k}}$ have relatively simple 
analytic forms, and can be compactly written as

$$
E_{\lambda \mathbf{k}}=s_{\lambda} \sqrt{\xi_{\mathbf{k}}^{2}+h^{2}+|\Delta|^{2}+\left|S_{\mathbf{k}}^{\perp}\right|^{2}+2 p_{\lambda} Z_{\mathbf{k}}},
$$

where $s_{1,3}=p_{3,4}=+1$ denote the quasiparticle and $s_{2,4}=$ $p_{1,2}=-1$ denote the quasihole branches which are particle-hole symmetric around zero energy, and $Z_{\mathbf{k}}=$ $\sqrt{\left(\xi_{\mathbf{k}}^{2}+|\Delta|^{2}\right) h^{2}+\left|S_{\mathbf{k}}^{\perp}\right|^{2} \xi_{\mathbf{k}}^{2}}$.

Given $\Omega$, the self-consistency equations are simply obtained by imposing the following conditions: $\partial \Omega / \partial|\Delta|=0$ gives an expression for the order parameter, $\partial \Omega / \partial \mu=-N$ gives the total number of $\uparrow$ and $\downarrow$ atoms, and $\partial \Omega / \partial h=-P N$ determines their polarization. This procedure leads to

$$
\begin{gathered}
\frac{2 M|\Delta|}{g}=\frac{1}{4} \sum_{\lambda \mathbf{k}} \frac{\partial E_{\lambda \mathbf{k}}}{\partial|\Delta|}\left(X_{\lambda \mathbf{k}}-1\right), \\
N_{\uparrow}+N_{\downarrow}=\frac{1}{4} \sum_{\lambda \mathbf{k}}\left[1+\frac{\partial E_{\lambda \mathbf{k}}}{\partial \mu}\left(X_{\lambda \mathbf{k}}-1\right)\right], \\
N_{\uparrow}-N_{\downarrow}=\frac{1}{4} \sum_{\lambda \mathbf{k}} \frac{\partial E_{\lambda \mathbf{k}}}{\partial h}\left(X_{\lambda \mathbf{k}}-1\right),
\end{gathered}
$$

where the derivatives are $\partial E_{\lambda \mathbf{k}} / \partial|\Delta|=\left(1+p_{\lambda} h^{2} / Z_{\mathbf{k}}\right)|\Delta| /$ $E_{\lambda \mathbf{k}}$ for the order parameter, $\partial E_{\lambda \mathbf{k}} / \partial \mu=-\left[1+p_{\lambda}\left(h^{2}+\right.\right.$ $\left.\left.\left|S_{\mathbf{k}}^{\perp}\right|^{2}\right) / Z_{\mathbf{k}}\right] \xi_{\mathbf{k}} / E_{\lambda \mathbf{k}}$ for the chemical potential, and $\partial E_{\lambda \mathbf{k}} / \partial h=$ $-\left[1+p_{\lambda}\left(\xi_{\mathbf{k}}^{2}+|\Delta|^{2}\right) / Z_{\mathbf{k}}\right] h / E_{\lambda \mathbf{k}}$ for the Zeeman field. Since $|\Delta|, \mu$ and $h$ are coupled for a given $t^{\prime}, g, \alpha, \beta$, and $T$, Eqs. (9)(11) need to be solved simultaneously for a self-consistent solution. Except for some limiting cases, the best way to achieve such solutions is through numerical means, e.g., via an iterative approach as discussed below in Sec. IV.

\section{TOPOLOGICAL PHASE TRANSITIONS}

Since it is possible to classify and distinguish all of the SF phases by the k-space topology of their zero-energy quasiparticle-quasihole excitations [25], next we analyze $E_{\lambda \mathbf{k}}$ for gapped and gapless solutions and gain some analytical insight before plunging into the numerical results.

\section{A. Gapless superfluids}

Equation (8) clearly shows that while $E_{3 \mathbf{k}}$ and $E_{4 \mathbf{k}}$ never vanish and are always gapped as long as $|\Delta| \neq 0, E_{1 \mathbf{k}}$ and $E_{2 \mathbf{k}}$ may vanish and can become gapless at some special $\mathbf{k}_{\mathbf{0}}$ points in $\mathbf{k}$ space. After a little bit of algebra, it can be easily verified that the condition $E_{1(2) \mathbf{k}}=0$ boils down to two conditions: $\left|S_{\mathbf{k}}^{\perp}\right|=0$ together with $\left(\xi_{\mathbf{k}}+h\right)\left(\xi_{\mathbf{k}}-h\right)+|\Delta|^{2}=0$. Therefore, while the number and locations of $\mathbf{k}_{\mathbf{0}}$ depend only on $t^{\prime}, \alpha$, and $\beta$, the critical Zeeman fields required $h_{\mathbf{k}_{0}}=\sqrt{\xi_{\mathbf{k}_{0}}^{2}+|\Delta|^{2}}$ depend on $t^{\prime}, \alpha, \beta, \epsilon_{\mathbf{k}_{0}}, \mu$, and $g$. Note that the presence of a SOC is not one of the essential ingredients that give rise to quantum phase transitions between SF phases with distinct $\mathbf{k}$-space topologies, and that these transitions are solely driven by $h \geqslant h_{\mathbf{k}_{0}}$ causing eventually $P \neq 0$ even in its absence.

For instance, we may have up to five sets of $\mathbf{k}_{\mathbf{0}}$ points satisfying the first gapless condition $\left|S_{\mathbf{k}_{0}}^{\perp}\right|=0$, which is discussed at length in Sec. IIC. Based on this analysis, it seems possible to have quantum phase transitions between SF phases with distinct k-space topologies at five different $h_{\mathbf{k}_{\mathbf{0}}}$ values. However, these distinct gapless-SF phases may only be realized assuming $|\Delta| \neq 0$ still pertains at sufficiently high Zeeman fields satisfying simultaneously the second gapless condition $h=h_{\mathbf{k}_{0}}$. The verification of this condition has to wait until Sec. IV, where we present our self-consistent solutions.

\section{B. Topological superfluids}

In the presence of an SOC, in addition to the gapped-gapless nature of $E_{\lambda \mathbf{k}}$ and the $\mathbf{k}$-space topology of the zero-energy quasiparticle-quasihole excitations, SF phases may further be characterized as topological or not depending on the integer value of the topological invariant of the system. By definition, a topologically invariant quantity may only change due to a change in the topology of the system, e.g., by opening or closing of an energy gap in $\mathbf{k}$ space, and therefore, the lowestenergy excitations of the system play a crucial role. To keep track of the possible changes in the topological invariant across the critical field $h_{\mathbf{k}_{0}}$, here we follow Bellissard's proposal [26] offering a simple and transparent way of calculating the changes in the $\mathrm{CN}$.

For this purpose, we reduce the $4 \times 4$ matrix $\mathbf{D}_{\mathbf{k}}$ to an effective $2 \times 2$ matrix $\mathbf{D}_{\mathbf{k k}_{0}}^{\text {eff }}$, describing the Hamiltonian dynamics in the neighborhood of $\mathbf{k}=\mathbf{k}_{\mathbf{0}}$ and $h=h_{\mathbf{k}_{0}}$. First, setting $\mathbf{k}=\mathbf{k}_{\mathbf{0}}$ and $S_{\mathbf{k}_{\mathbf{0}}}^{\perp}=0$ in $\mathbf{D}_{\mathbf{k}}$, we obtain much simpler expressions $E_{1(2) \mathbf{k}_{0}}= \pm\left(h_{\mathbf{k}_{0}}-h\right)$ for the $\lambda=\{1,2\}$ and $E_{3(4) \mathbf{k}_{0}}= \pm\left(h_{\mathbf{k}_{0}}+h\right)$ for the $\lambda=\{3,4\}$ branches of the quasiparticle-quasihole symmetric excitation spectrum. Then, the corresponding eigenvectors $\mathbf{f}_{\lambda_{\mathbf{k}_{0}}}$ for the zero-energy eigenvalues can be written as $\mathbf{f}_{1 \mathbf{k}_{0}}^{\mathrm{T}}=C_{1 \mathbf{k}_{\mathbf{0}}}\left(\frac{h_{\mathbf{k}_{0}}+\xi_{\mathbf{k}_{0}}}{\Delta^{*}}, 0,0,1\right)^{\mathrm{T}}$ for $E_{1 \mathbf{k}_{0}}=$ 0 and $\mathbf{f}_{2 \mathbf{k}_{0}}^{\mathrm{T}}=C_{2 \mathbf{k}_{0}}\left(0, \frac{h_{\mathbf{k}_{0}}-\xi_{\mathbf{k}_{0}}}{\Delta^{*}}, 1,0\right)^{\mathrm{T}}$ for $E_{2 \mathbf{k}_{0}}=0$. Here, $\mathrm{T}$ is the transposition operator, and $C_{1(2) \mathbf{k}_{0}}=|\Delta| / \sqrt{2 h_{\mathbf{k}_{0}}\left(h_{\mathbf{k}_{\mathbf{0}}} \pm \xi_{\mathbf{k}_{0}}\right)}$ are the normalization factors. Finally, projecting $\mathbf{D}_{\mathbf{k}}$ onto the two-dimensional zero-energy subspace of $\mathbf{f}_{1 \mathbf{k}_{0}}$ and $\mathbf{f}_{2 \mathbf{k}_{0}}$ eigenvectors gives the approximate Hamiltonian matrix $\mathbf{D}_{\mathbf{k k}_{\mathbf{0}}}^{\text {eff }}$ near the zeros, whose elements are $D_{\mathbf{k k}_{\mathbf{0}}}^{i j}=\mathbf{f}_{i \mathbf{k}_{\mathbf{0}}}^{\dagger} \mathbf{D}_{\mathbf{k}} \mathbf{f}_{j \mathbf{k}_{\mathbf{0}}}$. Therefore, $\mathbf{D}_{\mathbf{k k}_{\mathbf{0}}}^{\text {eff }}$ can be compactly written as $\mathbf{D}_{\mathbf{k k}_{\mathbf{0}}}^{\text {eff }}=d_{0} \sigma_{0}+\mathbf{d}_{\mathbf{k k}_{\mathbf{0}}} \cdot \vec{\sigma}$, where $d_{0}=0$ due to quasiparticle-quasihole symmetry, and the elements of the vector $\mathbf{d}_{\mathbf{k k}_{\mathbf{0}}}=\left(d_{\mathbf{k} \mathbf{k}_{\mathbf{0}}}^{x}, d_{\mathbf{k} \mathbf{k}_{\mathbf{0}}}^{y}, d_{\mathbf{k} \mathbf{k}_{\mathbf{0}}}^{z}\right)$ are

$$
\begin{gathered}
d_{\mathbf{k k}_{\mathbf{0}}}^{x}=\frac{|\Delta|}{h_{\mathbf{k}_{0}}} S_{\mathbf{k}}^{x}, \\
d_{\mathbf{k k}_{\mathbf{0}}}^{y}=\frac{|\Delta|}{h_{\mathbf{k}_{0}}} S_{\mathbf{k}}^{y}, \\
d_{\mathbf{k k}_{\mathbf{0}}}^{z}=\frac{|\Delta|^{2}}{h_{\mathbf{k}_{\mathbf{0}}}}\left(1-\frac{h h_{\mathbf{k}_{0}}-\xi_{\mathbf{k}} \xi_{\mathbf{k}_{0}}}{|\Delta|^{2}}\right) .
\end{gathered}
$$

These expressions satisfy [26] $d_{0}=\left(E_{1 \mathbf{k}}+E_{2 \mathbf{k}}\right) / 2$ and $\left|\mathbf{d}_{\mathbf{k k}_{\mathbf{0}}}\right| \approx\left(E_{1 \mathbf{k}}-E_{2 \mathbf{k}}\right) / 2$ in such a way that $\mathbf{d}_{\mathbf{k k}_{\mathbf{0}}}$ vanishes precisely at the locations of zeros.

According to Bellissard's proposal [26], the change in the $\mathrm{CN}$ at $h=h_{\mathbf{k}_{0}}$ is given by a sum over the Berry indices at all touching points,

$$
\begin{gathered}
\Delta \mathrm{CN}\left(h_{\mathbf{k}_{\mathbf{0}}}\right)=\sum_{\mathbf{k}_{\mathbf{0}}} \operatorname{sign}\left\{\operatorname{det}\left[\mathbf{J}_{\mathbf{f}}\left(\mathbf{k}_{\mathbf{0}}\right)\right]\right\}, \\
\operatorname{det}\left[\mathbf{J}_{\mathbf{f}}\left(\mathbf{k}_{\mathbf{0}}\right)\right]=\left[\frac{\partial d_{\mathbf{k k}_{\mathbf{0}}}^{x}}{\partial k_{x}} \frac{\partial d_{\mathbf{k k}_{\mathbf{0}}}^{y}}{\partial k_{y}}-\frac{\partial d_{\mathbf{k k}_{\mathbf{0}}}^{y}}{\partial k_{x}} \frac{\partial d_{\mathbf{k k}_{\mathbf{0}}}^{x}}{\partial k_{y}}\right]_{\mathbf{k}_{\mathbf{0}}}
\end{gathered}
$$


From the topological perspective, the unit vector $\widehat{\mathbf{d}}_{\mathbf{k k}_{0}}$ maps $\mathbf{k}$ space $\left(T^{2}\right)$ to an infinitesimally small unit sphere $\left(S^{2}\right)$ enclosing the zero at $\mathbf{k}_{\mathbf{0}}$, and $\mathbf{J}_{\mathbf{f}}\left(\mathbf{k}_{\mathbf{0}}\right)$ is the Jacobian matrix for the corresponding transformation. The $\mathrm{CN}$ is physically the number of times that $\widehat{\mathbf{d}}_{\mathbf{k k}_{0}}$ winds around $S^{2}$ as $\mathbf{k}$ varies, and therefore, it is also called the winding number. Note that, since $\partial d_{\mathbf{k k}_{\mathbf{0}}}^{x} / \partial h=0, \partial d_{\mathbf{k k}_{\mathbf{0}}}^{y} / \partial h=0$ and $\partial d_{\mathbf{k k}_{\mathbf{0}}}^{z} / \partial h=-1$ in our problem, the Jacobian of the transformation satisfies $\operatorname{det}\left[\mathbf{J}_{\mathbf{f}}\left(h_{0}, \mathbf{k}_{\mathbf{0}}\right)\right]=-\operatorname{det}\left[\mathbf{J}_{\mathbf{f}}\left(\mathbf{k}_{\mathbf{0}}\right)\right]$.

For our square lattice, using Eqs. (4) and (5) in Eq. (16), we find

$$
\begin{aligned}
\operatorname{det}\left[\mathbf{J}_{\mathbf{f}}\left(\mathbf{k}_{\mathbf{1}}\right)\right]= & \frac{4|\Delta|^{2} t^{2}}{h_{\mathbf{k}_{\mathbf{1}}}^{2}}\left[\sin \alpha \sin \beta+4 \alpha \beta \frac{\sin ^{2} \gamma}{\gamma^{2}} \frac{t^{\prime 2}}{t^{2}}\right. \\
& \left.+2(\alpha \sin \beta+\beta \sin \alpha) \frac{\sin \gamma}{\gamma} \frac{t^{\prime}}{t}\right]
\end{aligned}
$$

for the first set of zeros. Simply substitute $\sin \alpha \rightarrow-\sin \alpha$ in this expression to obtain $\operatorname{det}\left[\mathbf{J}_{\mathbf{f}}\left(\mathbf{k}_{2}\right)\right]$, substitute $\sin \beta \rightarrow$ $-\sin \beta$ to obtain $\operatorname{det}\left[\mathbf{J}_{\mathbf{f}}\left(\mathbf{k}_{\mathbf{3}}\right)\right]$, and substitute $\sin \alpha \rightarrow-\sin \alpha$ together with $\sin \beta \rightarrow-\sin \beta$ to obtain $\operatorname{det}\left[\mathbf{J}_{\mathbf{f}}\left(\mathbf{k}_{\mathbf{4}}\right)\right]$. For the last set of zeros, we likewise obtain

$$
\begin{aligned}
\operatorname{det}\left[\mathbf{J}_{\mathbf{f}}\left(\mathbf{k}_{\mathbf{5}}\right)\right]= & -\frac{16|\Delta|^{2} t^{\prime 2}}{h_{\mathbf{k}_{\mathbf{5}}}^{2}} \alpha \beta\left(\frac{\sin ^{2} \gamma}{\gamma^{2}}-\frac{\sin ^{2} \beta}{4 \beta^{2}} \frac{t^{2}}{t^{\prime 2}}\right) \\
& \times\left(\frac{\sin ^{2} \gamma}{\gamma^{2}}-\frac{\sin ^{2} \alpha}{4 \alpha^{2}} \frac{t^{2}}{t^{\prime 2}}\right),
\end{aligned}
$$

which is always negative when $\mathbf{k}_{\mathbf{5}}$ is relevant. Therefore, $\Delta \mathrm{CN}\left(h_{\mathbf{k}_{5}}\right)=-4$ for all parameters as long as $\left|t^{\prime}\right| / t$ is beyond the critical threshold value given in Sec. II C. Since the total change in the $\mathrm{CN}$ must add up to zero in the normal phase when $h \gg t$, this suggests that $\Delta \mathrm{CN}\left(h_{\mathbf{k}_{1}}\right)=$ $\Delta \mathrm{CN}\left(h_{\mathbf{k}_{2}}\right)=\Delta \mathrm{CN}\left(h_{\mathbf{k}_{3}}\right)=\Delta \mathrm{CN}\left(h_{\mathbf{k}_{4}}\right)=+1$ for all parameters, when $\mathbf{k}_{\mathbf{5}}$ is relevant. This is best revealed in the following limits.

(i) When $t^{\prime}=0$, Eq. (17) reduces to $\operatorname{det}\left[\mathbf{J}_{\mathbf{f}}\left(\mathbf{k}_{\mathbf{1}}\right)\right]=$ $-\operatorname{det}\left[\mathbf{J}_{\mathbf{f}}\left(\mathbf{k}_{\mathbf{2}}\right)\right]=-\operatorname{det}\left[\mathbf{J}_{\mathbf{f}}\left(\mathbf{k}_{\mathbf{3}}\right)\right]=\operatorname{det}\left[\mathbf{J}_{\mathbf{f}}\left(\mathbf{k}_{\mathbf{4}}\right)\right]=4|\Delta|^{2} t^{2} \sin \alpha$ $\sin \beta / h_{\mathbf{k}_{123,4}}^{2}$, for which the corresponding energy dispersions are given by $\epsilon_{\mathbf{k}_{1}}=-\epsilon_{\mathbf{k}_{4}}=-2 t(\cos \alpha+\cos \beta)$ and $\epsilon_{\mathbf{k}_{2}}=-\epsilon_{\mathbf{k}_{3}}=-2 t(\cos \alpha-\cos \beta)$. Note that $\mathbf{k}_{\mathbf{5}}$ is irrelevant in this limit. Therefore, $\Delta \mathrm{CN}\left(h_{\mathbf{k}_{1}}\right)=\Delta \mathrm{CN}\left(h_{\mathbf{k}_{4}}\right)=+1$ and $\Delta \mathrm{CN}\left(h_{\mathbf{k}_{2}}\right)=\Delta \mathrm{CN}\left(h_{\mathbf{k}_{3}}\right)=-1$. However, $h_{\mathbf{k}_{0}}$ are fourfold (twofold) degenerate when $\alpha=\pi / 2$ and (or) $\beta=\pi / 2$ with opposite contributions to the change in $\mathrm{CN}$, and therefore, the total change in the $\mathrm{CN}$ vanishes for any $\mu$.

(ii) When $t=0$, Eqs. (17) and (18) reduce to $\operatorname{det}\left[\mathbf{J}_{\mathbf{f}}\left(\mathbf{k}_{\mathbf{1}}\right)\right]=$ $\operatorname{det}\left[\mathbf{J}_{\mathbf{f}}\left(\mathbf{k}_{\mathbf{2}}\right)\right]=\operatorname{det}\left[\mathbf{J}_{\mathbf{f}}\left(\mathbf{k}_{\mathbf{3}}\right)\right]=\operatorname{det}\left[\mathbf{J}_{\mathbf{f}}\left(\mathbf{k}_{\mathbf{4}}\right)\right]=-\operatorname{det}\left[\mathbf{J}_{\mathbf{f}}\left(\mathbf{k}_{\mathbf{5}}\right)\right]=$ $16|\Delta|^{2} \alpha \beta \sin ^{2} \gamma t^{\prime 2} /\left(\gamma^{2} h_{\mathbf{k}_{1,2,3,4}}^{2}\right)$, for which the corresponding energy dispersions are given by $\epsilon_{\mathbf{k}_{1}}=-\epsilon_{\mathbf{k}_{2}}=-\epsilon_{\mathbf{k}_{3}}=$ $\epsilon_{\mathbf{k}_{4}}=-4 t^{\prime} \cos \gamma$ and $\epsilon_{\mathbf{k}_{5}}=0$. When $\mu \neq 0$, the fields $h_{\mathbf{k}_{1}}=$ $h_{\mathbf{k}_{4}}$ and $h_{\mathbf{k}_{2}}=h_{\mathbf{k}_{3}}$ are twofold degenerate, and therefore, $\Delta \mathrm{CN}\left(h_{\mathbf{k}_{1,4}}\right)=\Delta \mathrm{CN}\left(h_{\mathbf{k}_{2,3}}\right)=+2$ and $\Delta \mathrm{CN}\left(h_{\mathbf{k}_{5}}\right)=-4$. At $\mu=0$, however, $h_{\mathbf{k}_{\mathbf{0}}}$ are fourfold degenerate, and $\Delta \mathrm{CN}$ changes from 0 to -4 to 0 as $h$ increases from 0 to $h_{\mathbf{k}_{5}}=|\Delta|$ to $h_{\mathbf{k}_{1,2,3,4}}=\sqrt{16 t^{\prime 2} \cos ^{2} \gamma+|\Delta|^{2}}$.

(iii) When $\alpha=\beta$, Eq. (17) reduces to $\operatorname{det}\left[\mathbf{J}_{\mathbf{f}}\left(\mathbf{k}_{\mathbf{1}}\right)\right]=$ $4|\Delta|^{2}\left[t \sin \alpha+\sqrt{2} t^{\prime} \sin (\sqrt{2} \alpha)\right]^{2} / h_{\mathbf{k}_{1}}^{2}$, and we find det
$\left[\mathbf{J}_{\mathbf{f}}\left(\mathbf{k}_{\mathbf{2}}\right)\right]=\operatorname{det}\left[\mathbf{J}_{\mathbf{f}}\left(\mathbf{k}_{\mathbf{3}}\right)\right]=-4|\Delta|^{2}\left[t^{2} \sin ^{2} \alpha-2 t^{\prime 2} \sin ^{2}(\sqrt{2} \alpha)\right] /$ $h_{\mathbf{k}_{2,3}}^{2}$ for the second and third sets of zeros, and $\operatorname{det}\left[\mathbf{J}_{\mathbf{f}}\left(\mathbf{k}_{\mathbf{4}}\right)\right]=$ $4|\Delta|^{2}\left[t \sin \alpha-\sqrt{2} t^{\prime} \sin (\sqrt{2} \alpha)\right]^{2} / h_{\mathbf{k}_{4}}^{2}$ for the fourth set of zeros. In addition, Eq. (18) reduces to $\operatorname{det}\left[\mathbf{J}_{\mathbf{f}}\left(\mathbf{k}_{\mathbf{5}}\right)\right]=$ $-8|\Delta|^{2}\left[\frac{\sin ^{2} \alpha}{\sin (\sqrt{2} \alpha)} \frac{t^{2}}{2 t^{\prime}}-\sin (\sqrt{2} \alpha) t^{\prime}\right]^{2} / h_{\mathbf{k}_{5}}^{2}$ which is relevant for $\left|t^{\prime}\right|>t \sin \alpha /[\sqrt{2} \sin (\sqrt{2} \alpha)]$. The corresponding energy dispersions are given by $\epsilon_{\mathbf{k}_{1}}=-4 t \cos \alpha-4 t^{\prime} \cos (\sqrt{2} \alpha), \epsilon_{\mathbf{k}_{2}}=$ $\epsilon_{\mathbf{k}_{3}}=4 t^{\prime} \cos (\sqrt{2} \alpha), \epsilon_{\mathbf{k}_{4}}=4 t \cos \alpha-4 t^{\prime} \cos (\sqrt{2} \alpha)$, and $\epsilon_{\mathbf{k}_{5}}=$ $\sqrt{2} \frac{t^{2}}{t^{\prime}}\left[\frac{\sin (2 \alpha)}{\sin (\sqrt{2} \alpha)}-\frac{\sqrt{2} \sin ^{2} \alpha}{\sin (\sqrt{2} \alpha) \tan (\sqrt{2} \alpha)}\right]$. Therefore, since $h_{\mathbf{k}_{2}}=h_{\mathbf{k}_{3}}$ is twofold degenerate, we find $\Delta \mathrm{CN}\left(h_{\mathbf{k}_{1}}\right)=\Delta \mathrm{CN}\left(h_{\mathbf{k}_{4}}\right)=$ $+1, \Delta \mathrm{CN}\left(h_{\mathbf{k}_{2,3}}\right)= \pm 2$, and $\Delta \mathrm{CN}\left(h_{\mathbf{k}_{5}}\right)=-4$, depending on whether $\mathbf{k}_{\mathbf{5}}$ is relevant or not.

\section{NUMERICAL RESULTS}

As we discussed thoroughly in the previous section, it is possible to have quantum phase transitions between $\mathrm{SF}$ phases with distinct k-space topologies, assuming $|\Delta| \neq 0$ still pertains at sufficiently high Zeeman fields satisfying the condition $h=h_{\mathbf{k}_{0}}$. In this section, we solve self-consistency Eqs. (9)-(11) at $T=0$, and present the resultant ground-state phase diagrams as functions of total particle filling $F=$ $\left(N_{\uparrow}+N_{\downarrow}\right) / M$ and NNN hopping $t^{\prime} / t$ for various polarizations $P=\left(N_{\uparrow}-N_{\downarrow}\right) /\left(N_{\uparrow}+N_{\downarrow}\right)$, interaction strengths $g / t$, and SOC strengths $(\alpha, \beta)$. Note that, since $0 \leqslant N_{\sigma} / M \leqslant 1$ within the single-band Hubbard model used in this paper, $F$ has an upper bound for a given $P$ as $F_{\max }=2 /(1 \pm P)$ depending on $P \gtrless 0$, and that we assume $P \geqslant 0$ in this paper without losing generality. The normal phase is characterized by $|\Delta|<10^{-3} t$ in our numerical calculations.

First, we study the effects of polarization in Fig. 3, where we set $\alpha=\beta=\pi / 4$ and $g=10 t$, and show colored maps of $|\Delta| / t$ and the corresponding boundaries for the topological quantum phase transitions as functions of $0 \leqslant$ $F \leqslant 2 /\left(1+P\right.$ ) and $t^{\prime} / t$ for (a) $P=0.1$, (b) 0.3 , (c) 0.4 , and (d) 0.7 . The SF phase is always gapped, and therefore, it is topologically trivial for low Zeeman fields as long as $h<h_{\mathbf{k}_{\mathbf{0}}}$ for all $\mathbf{k}_{\mathbf{0}}$ or equivalently $P=0$. However, the intricate dependence of DoS on $\omega$ and $t^{\prime}$ shown in Fig. 2 gives rise to an equivalently intricate dependence of $|\Delta|$ on $0 \leqslant F \leqslant 2$ and $t^{\prime}$. That is, not only the $D\left(\omega, t^{\prime}\right)=D\left(-\omega,-t^{\prime}\right)$ symmetry causes $\left|\Delta\left(F, t^{\prime}\right)\right|=\left|\Delta\left(2-F,-t^{\prime}\right)\right|$, but also $|\Delta|$ is pronounced and suppressed whenever $D(\omega)$ is pronounced and suppressed as intuitively expected from the BCS theory. For instance, in the absence of an SOC, the peak value of $|\Delta|$ first shifts from $F=1$ to higher (lower) values as $t^{\prime}$ increases (decreases) from 0 to $0.5 t(-0.5 t)$, and then it gradually returns back to $F=1$ in the $\left|t^{\prime}\right| \gg t$ limit. In the presence of an SOC, however, the narrow strips in $D(\omega)$ causes multiple reentrant SF phases, separated by the fingering normal phase, as a function of $F$.

Even though we do not present $|\Delta|$ in the strict $P=0$ limit, remnants of the reentrant SF phases are clearly seen in our $P \neq 0$ figures. Note that since increasing $P$ dramatically weakens $|\Delta|$ for a given $t^{\prime} / t$, additional features of $D(\omega)$ shown in Fig. 2(c) vaguely appear in Fig. 3(d) but somewhat hindered by the dominant effects of strong $g / t$. In addition to the direct connection between $D(\omega)$ and $|\Delta|$, these figures also illustrate how topological phase transition boundaries 
(a)

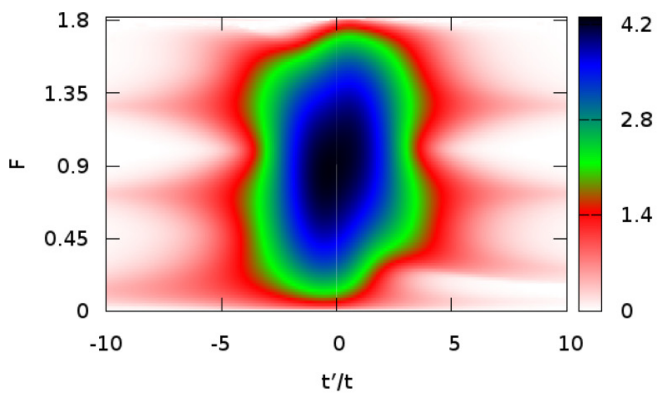

(b)

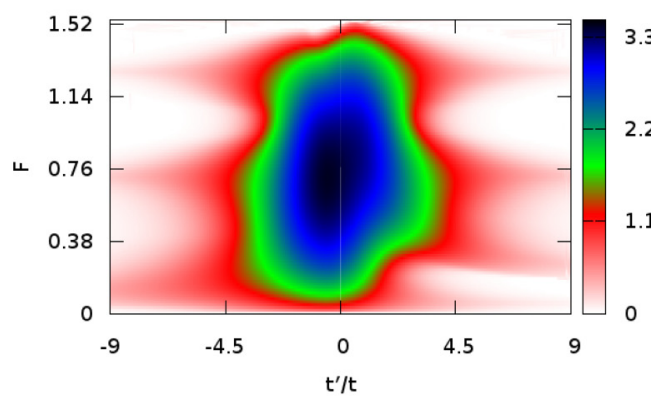

(c)

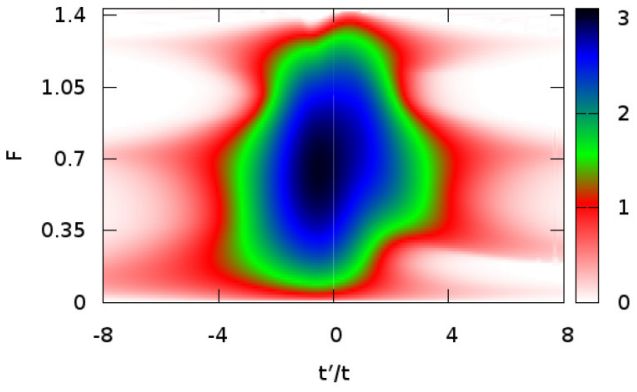

(d)

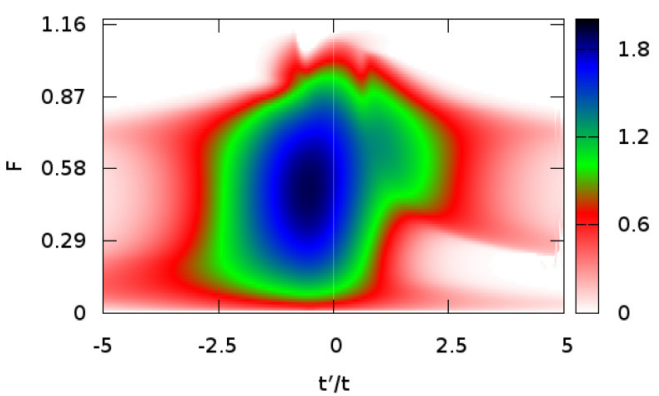

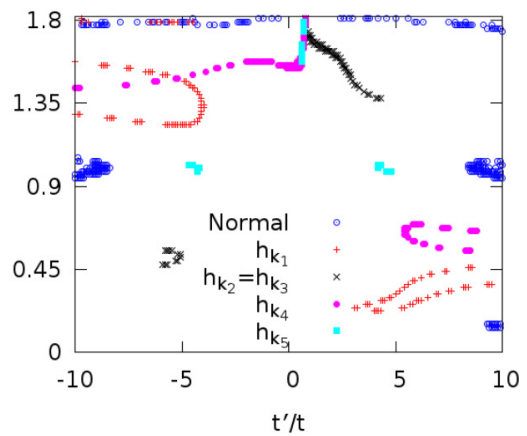
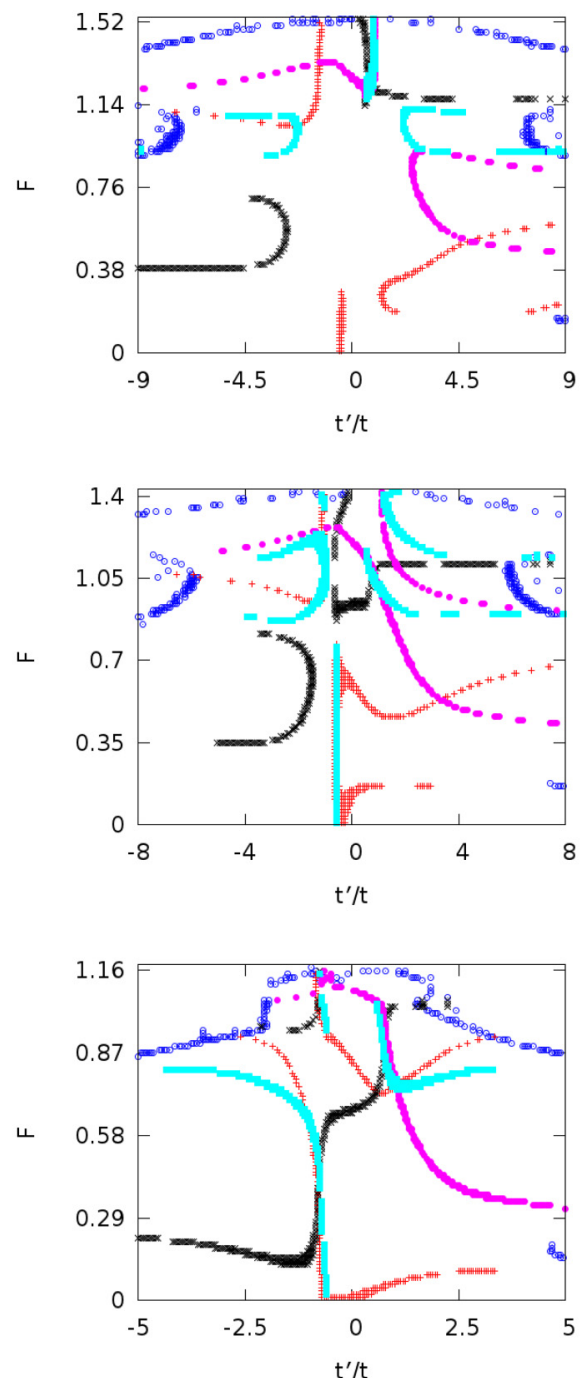

FIG. 3. The colored maps of the SF order parameter $|\Delta| / t$ are shown as functions of total particle filling $F$ and NNN hopping $t^{\prime} / t$ for (a) $P=0.1$, (b) 0.3 , (c) 0.4 , and (d) 0.7 , where $\alpha=\beta=\pi / 4$ and $g=10 t$. The corresponding boundaries for the topological quantum phase transitions are determined by the condition $h=h_{\mathbf{k}_{i}}=\sqrt{\xi_{\mathbf{k}_{\mathbf{i}}}^{2}+|\Delta|^{2}}$, and they are also extracted and shown on the right panel.

gradually enter the stage as $P$ increases from 0 . Since a gapless-SF phase can be considered as a coexistence of gapped-SF and normal phases, we expect them to exist at the interfaces between gapped SF and normal phases. This is best illustrated in the $P=0.1$ phase diagram shown in Fig. 3(a), where the gapless-SF phases first emerge in the parameter regions where $|\Delta|$ is most weakest. The gapless-SF phases progressively occupy more and more territory in Figs. 3(b)3(d) with increasing $P$.

Similarly, we study the effects of interaction strength in Fig. 4, where we set $\alpha=\beta=\pi / 4$ and $P=0.5$, and show colored maps of $|\Delta| / t$ and the corresponding boundaries for the topological quantum phase transitions as functions of $0 \leqslant F \leqslant 4 / 3$ and $t^{\prime} / t$ for (a) $g=4 t$, (b) $5 t$, (c) $7 t$, and (d) 
(a)

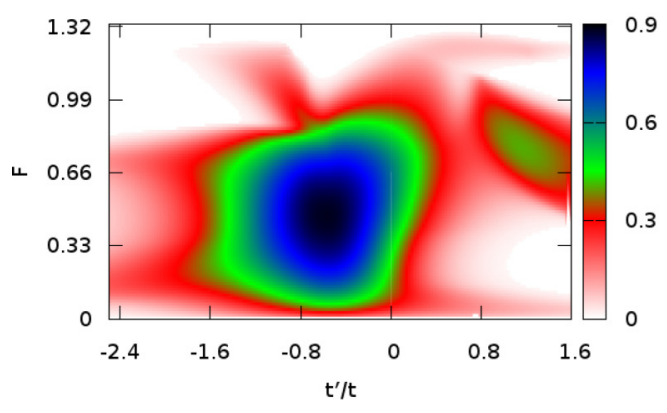

(b)

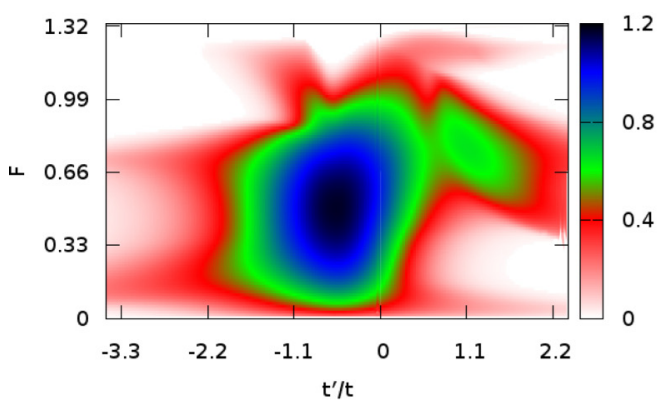

(c)

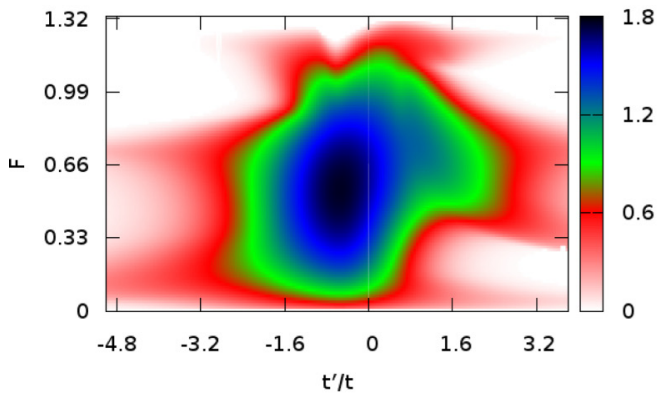

(d)

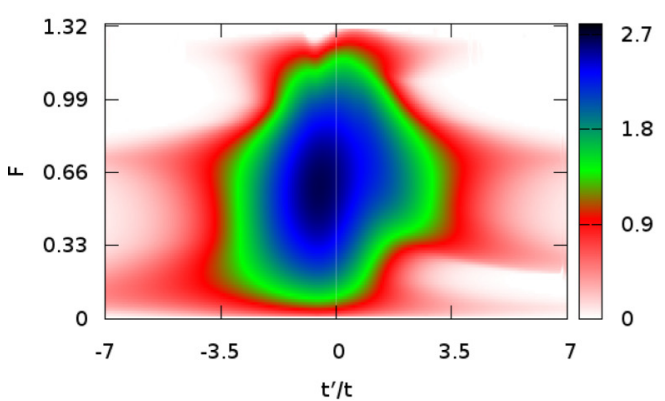

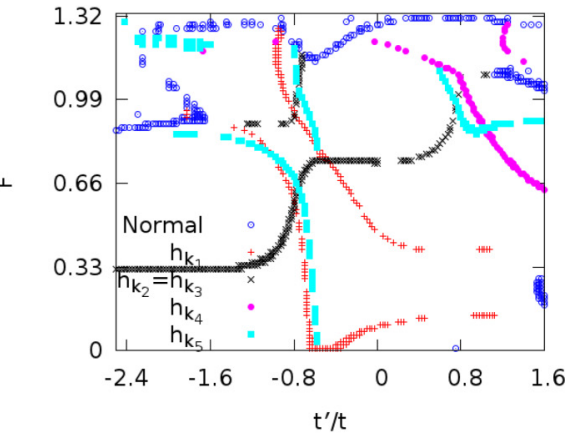
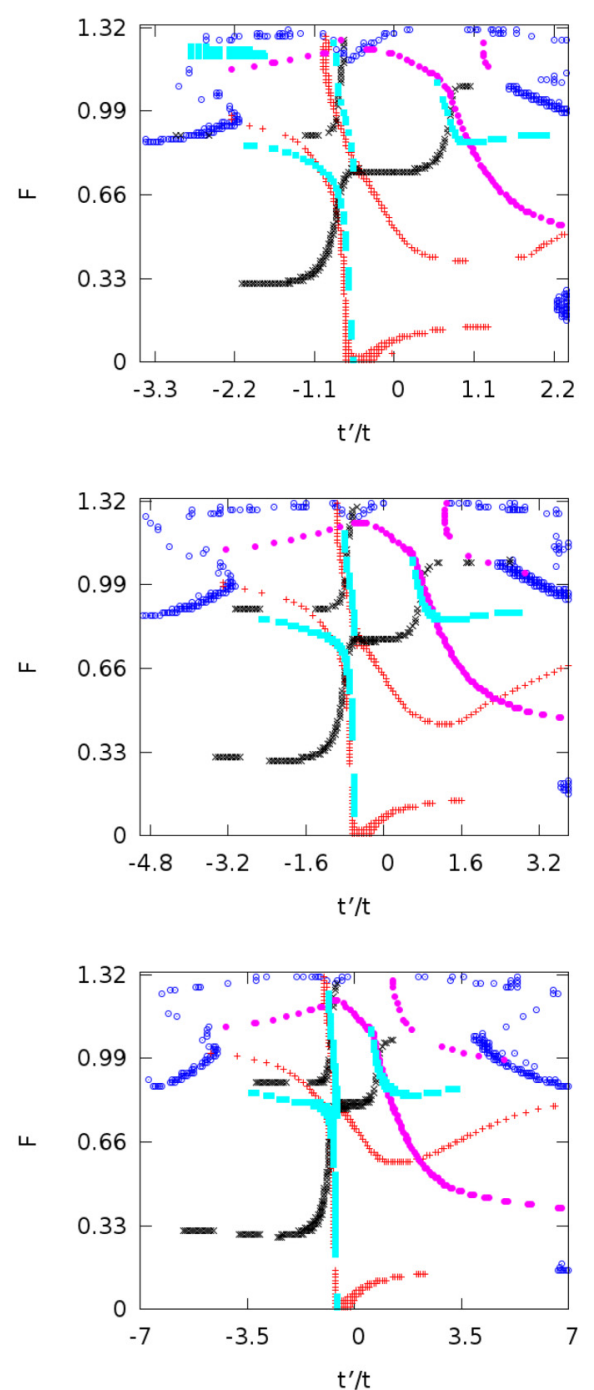

FIG. 4. The colored maps of the SF order parameter $|\Delta| / t$ are shown as functions of total particle filling $F$ and NNN hopping $t^{\prime} / t$ for (a) $g=4 t$, (b) $5 t$, (c) $7 t$, and (d) $10 t$, where $\alpha=\beta=\pi / 4$ and $P=0.5$. The corresponding boundaries for the topological quantum phase transitions are determined by the condition $h=h_{\mathbf{k}_{i}}=\sqrt{\xi_{\mathbf{k}_{\mathbf{i}}}^{2}+|\Delta|^{2}}$, and they are also extracted and shown on the right panel.

$10 t$. Note that, since $g / t$ is relatively weak, and therefore, $|\Delta|$ is considerably small in Figs. 4(a) and 4(b), additional features of $D(\omega)$ shown in Fig. 2(c) vaguely appear in these figures especially around $\left|t^{\prime}\right| \sim t$, but they are again somewhat disguised in Figs. 4(c) and 4(d) where stronger $g / t$ effects dominate.
Lastly, we study the effects of SOC strength in Fig. 5, where we set $g=10 t$ and $P=0.5$, and show colored maps of $|\Delta| / t$ and the corresponding boundaries for the topological quantum phase transitions as functions of $0 \leqslant F \leqslant 4 / 3$ and $t^{\prime} / t$ for (a) $\alpha=\beta=0$, (b) $\pi / 8$, (c) $\pi / 3$, and (d) $\pi / 2$. These figures further reveal the direct connection between 
(a)

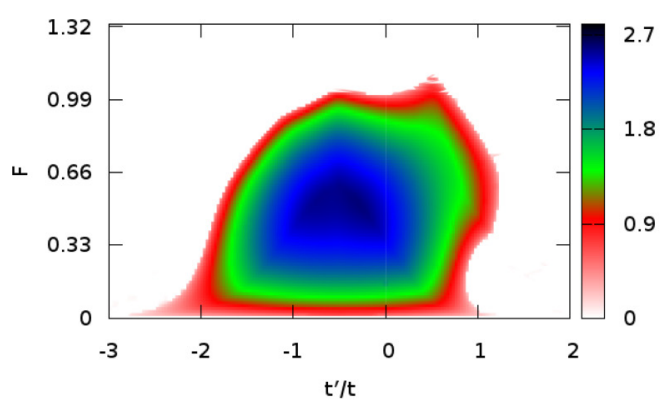

(b)

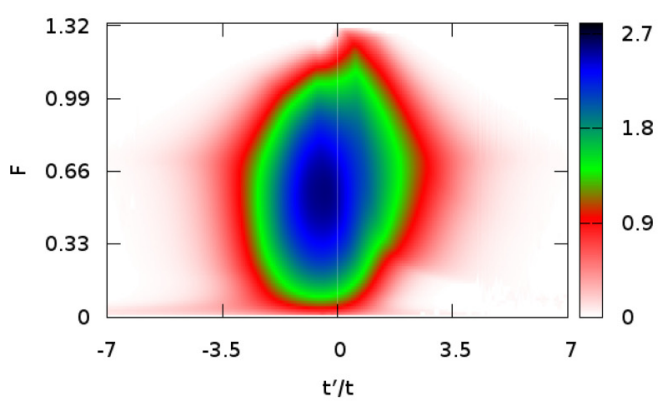

(c)

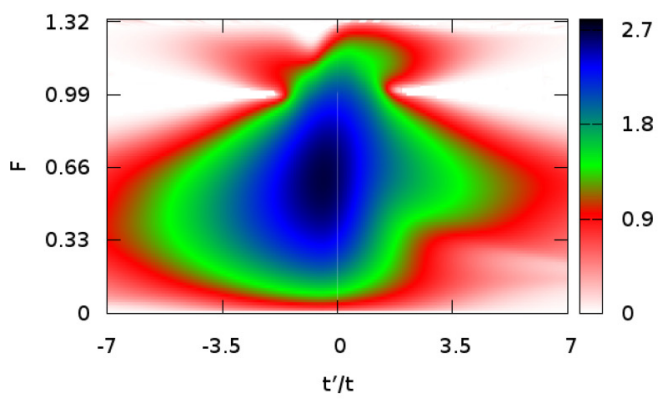

(d)

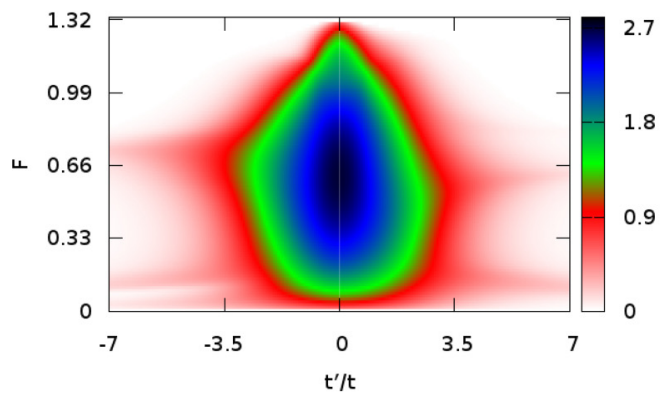

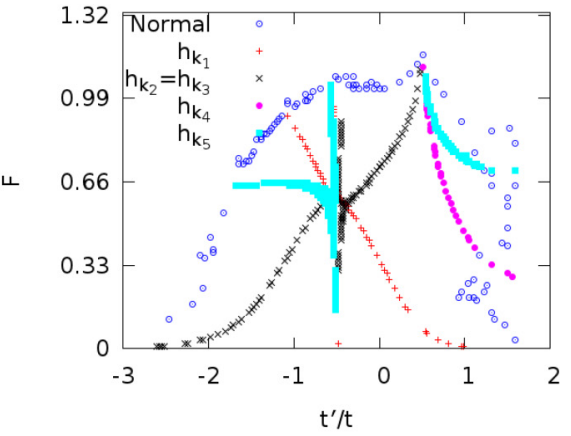
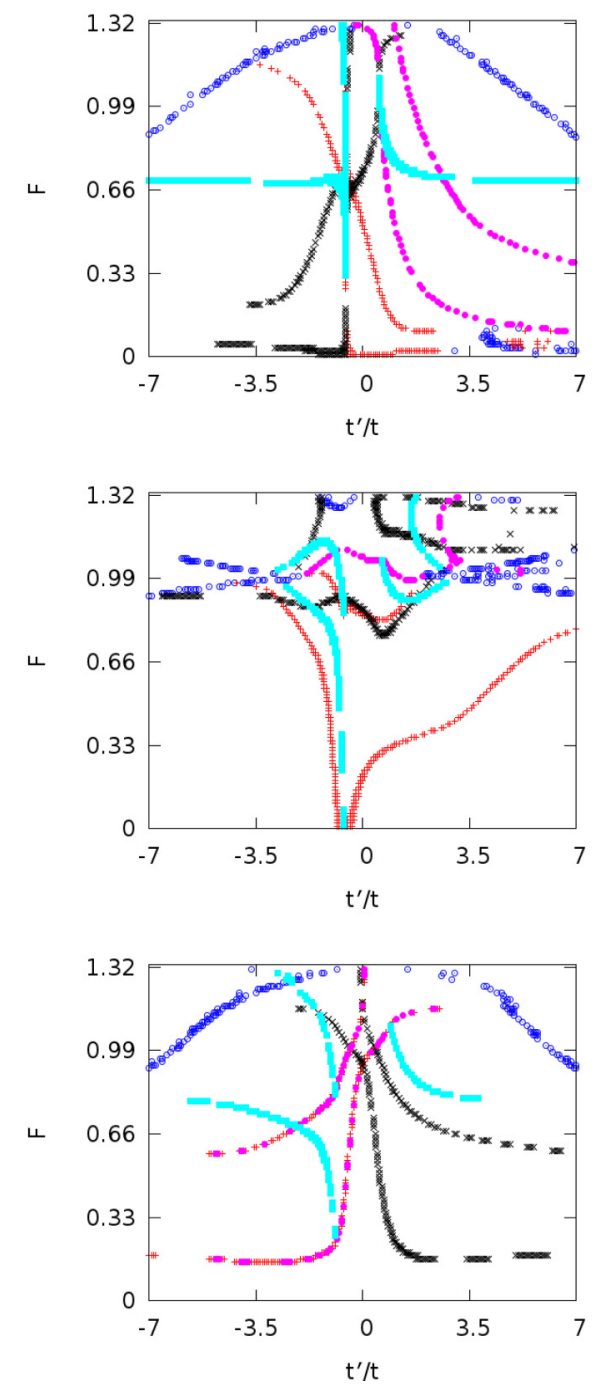

FIG. 5. The colored maps of the SF order parameter $|\Delta| / t$ are shown as functions of total particle filling $F$ and NNN hopping $t^{\prime} / t$ for (a) $\alpha=\beta \rightarrow 0^{+}$, (b) $\pi / 8$, (c) $\pi / 3$, and (d) $\pi / 2$, where $g=10 t$ and $P=0.5$. The corresponding boundaries for the topological quantum phase transitions are determined by the condition $h=h_{\mathbf{k}_{i}}=\sqrt{\xi_{\mathbf{k}_{\mathbf{i}}}^{2}+|\Delta|^{2}}$, and they are also extracted and shown on the right panel.

the $D\left(\omega, t^{\prime}\right)=D\left(-\omega,-t^{\prime}\right)$ symmetry and that of $\left|\Delta\left(F, t^{\prime}\right)\right|=$ $\left|\Delta\left(2-F,-t^{\prime}\right)\right|$ symmetry in spite of the apparent asymmetry of $|\Delta|$ caused by $P \neq 0$. For instance, while the reentrant structure ceases to exist and the SF phase occupies a compact territory in Fig. 5(a), it can still be seen in Figs. 5(b)-5(d) in spite of strong $g / t$. We emphasize that even though the presence of a SOC is indispensable for creating a topological-
SF phase, it is not one of the essential ingredients that bring about quantum phase transitions between SF phases with distinct k-space topologies. In the absence of an SOC, these transitions are solely driven by $h$ causing eventually $P \neq 0$, and they are clearly illustrated on the right panel in Fig. 5(a).

Given these numerical results, we firmly establish that a plethora of quantum phase transitions are possible between 
SF phases with distinct zero-energy quasiparticle-quasihole excitation topologies in $\mathbf{k}$ space in our model Hamiltonian given in Eq. (6). Having achieved our primary objective, we are ready to end the paper with a brief summary of our conclusions and an outlook.

\section{SUMMARY AND OUTLOOK}

To summarize, here we considered a two-component Fermi gas with attractive interactions on a square optical lattice, and studied the combined effects of Zeeman field, SOC, and NNN hopping on the ground-state phase diagrams in the entire BCS-BEC evolution. For this purpose, we first discussed the effects of SOC both on the single-particle problem and on the corresponding DoS, and derived the self-consistency mean-field equations for handling the many-body problem. We then classified and distinguished all possible SF phases by the k-space topology of their zero-energy quasiparticle-quasihole excitations, and numerically established that numerous quantum phase transitions are possible in between driven mainly by the Zeeman field. In addition, we also derived analytical expressions for the changes in the $\mathrm{CN}$ showing that these phase transitions are further signaled and evidenced by the changes in the topological invariant of the system when there is SOC.

In addition to these important results, we found that the SF phase exhibits a reentrant structure, separated by a fingering normal phase, as a function of total particle filling. This intricate structure is a result of combined SOC and NNN hopping, and we traced its origin back to the single-particle DoS of the system which is shown to exhibit a number of narrow strips (depending on the SOC strength) as the energy changes for a given NNN hopping. Given the ongoing experimental interest in spin-orbit coupled systems in various physics communities, even though we are aware of many technical difficulties in observing much of these results in the ongoing cold-atom experiments, we hope that some of their signatures may still be realized in the foreseeable future. For instance, while the presence of an SOC is crucial for creating both topological-SF phases and reentrant SF behavior, the aforementioned quantum phase transitions between SF phases with distinct k-space topologies are exclusively driven by the Zeeman field, and therefore, they can be observed in the vanishing SOC and/or NNN hopping limits as well. We, at least, believe that the rich physics involved in this simple model may shed some light and trigger further research in related problems.

As an outlook of this work, even though we expect competing SF phases such as the ones involving finite centerof-mass pairing to play a minor role in the presence of an SOC [25], the possibility of Fulde-Ferrell-Larkin-Ovchinnikov like SF phases, e.g., a topological Fulde-Ferrell SF [27-29], may still be explored in this model. Since such a task requires a much higher computing power, it is beyond the scope of this paper. Alternatively, instead of the k-space description employed here, one can use the real-space Bogoliubov-de Gennes description and include the finite-size effects caused by the trapping potential [30]. The latter is a fully numerical approach and it may not only provide a way more complete understanding of the competing SF phases (at a cost of less analytical insight into their nature), but also the fate of spontaneous mass and spin currents, polarization and spin textures, etc., can as well be investigated. Last but not least, our preliminary calculations on triangular lattices suggest that it may be valuable to extend this formalism to other kinds of two-dimensional geometries including the honeycomb lattice, an important direction that is highly feasible in the advent of tunable optical lattices [8,31].

\section{ACKNOWLEDGMENT}

We gratefully acknowledge funding from TÜBİTAK Grant No. 1001-114F232.
[1] T. Esslinger, Annu. Rev. Condens. Matter Phys. 1, 129 (2010).

[2] W. Beugeling, J. C. Everts, and C. Morais Smith, Phys. Rev. B 86, 195129 (2012).

[3] Jiao Miao, Phys. Rev. A 92, 023632 (2015).

[4] H. Lignier, C. Sias, D. Ciampini, Y. Singh, A. Zenesini, O. Morsch, and E. Arimondo, Phys. Rev. Lett. 99, 220403 (2007).

[5] J. Struck, C. Ölschläger, M. Weinberg, P. Hauke, J. Simonet, A. Eckardt, M. Lewenstein, K. Sengstock, and P. Windpassinger, Phys. Rev. Lett. 108, 225304 (2012).

[6] C. V. Parker, L.-C. Ha, and C. Chin, Nature Phys. 9, 769 (2013).

[7] F. D. M. Haldane, Phys. Rev. Lett. 61, 2015 (1988).

[8] G. Jotzu, M. Messer, R. Desbuquois, M. Lebrat, T. Uehlinger, D. Greif, and T. Esslinger, Nature (London) 515, 237 (2014).

[9] J. Dalibard, F. Gerbier, G. Juzelinas, and P. Öhberg, Rev. Mod. Phys. 83, 1523 (2011).

[10] V. Galitski and I. B. Spielman, Nature (London) 494, 49 (2013).

[11] M. Aidelsburger, M. Atala, M. Lohse, J. T. Barreiro, B. Paredes, and I. Bloch, Phys. Rev. Lett. 111, 185301 (2013).
[12] H. Miyake, G. A. Siviloglou, C. J. Kennedy, W. C. Burton, and W. Ketterle, Phys. Rev. Lett. 111, 185302 (2013).

[13] J.-Y. Zhang, S.-C. Ji, Z. Chen, L. Zhang, Z.-D. Du, B. Yan, G.-S. Pan, B. Zhao, Y.-J. Deng, H. Zhai, S. Chen, and J.-W. Pan, Phys. Rev. Lett. 109, 115301 (2012).

[14] P. Wang, Z.-Q. Yu, Z. Fu, J. Miao, L. Huang, S. Chai, H. Zhai, and J. Zhang, Phys. Rev. Lett. 109, 095301 (2012).

[15] L. W. Cheuk, A. T. Sommer, Z. Hadzibabic, T. Yefsah, W. S. Bakr, and M. W. Zwierlein, Phys. Rev. Lett. 109, 095302 (2012).

[16] C. Qu, C. Hamner, M. Gong, C. Zhang, and P. Engels, Phys. Rev. A 88, 021604(R) (2013).

[17] Z. Fu, L. Huang, Z. Meng, P. Wang, X.-J. Liu, H. Pu, H. Hu, and J. Zhang, Phys. Rev. A 87, 053619 (2013).

[18] R. A. Williams, M. C. Beeler, L. J. LeBlanc, K. Jiménez-García, and I. B. Spielman, Phys. Rev. Lett. 111, 095301 (2013).

[19] A. Kubasiak, P. Massignan, and M. Lewenstein, Europhys. Lett. 92, 46004 (2010). 
[20] A somewhat similar model Hamiltonian with purely real NNN hoppings is recently studied in a non-self-consistent fashion by R. Wang, Y. F. Zhang, and C. X. Zhang, Res. Phys. 4, 44 (2014).

[21] M. Feld, B. Frohlich, E. Vogt, M. Koschorreck, and M. Köhl, Nature (London) 480, 75 (2011).

[22] A. T. Sommer, L. W. Cheuk, M. J.-H. Ku, W. S. Bakr, and M. W. Zwierlein, Phys. Rev. Lett. 108, 045302 (2012).

[23] V. Makhalov, K. Martiyanov, and A. Turlapov, Phys. Rev. Lett. 112, 045301 (2014).

[24] M. G. Ries, A. N. Wenz, G. Zürn, L. Bayha, I. Boettcher, D. Kedar, P. A. Murthy, M. Neidig, T. Lompe, and S. Jochim, Phys. Rev. Lett. 114, 230401 (2015).
[25] M. Iskin and A. L. Subaşı, Phys. Rev. A 87, 063627 (2013).

[26] J. Bellissard, arXiv:cond-mat/9504030.

[27] Wei Zhang and Wei Yi, Nature Comm. 4, 2711 (2013).

[28] C. Qu, Z. Zheng, M. Gong, Y. Xu, L. Mao, X. Zou, G. Guo, and C. Zhang, Nature Comm. 4, 2710 (2013).

[29] X.-J. Liu, Hui Hu, and Han Pu, Chin. Phys. B 24, 050502 (2015).

[30] M. Iskin, Phys. Rev. A 88, 013631 (2013).

[31] T. Uehlinger, G. Jotzu, M. Messer, D. Greif, W. Hofstetter, U. Bissbort, and T. Esslinger, Phys. Rev. Lett. 111, 185307 (2013). 